\title{
Stability of ZrTiCuNiBe bulk metallic glass upon isothermal annealing near the glass transition temperature
}

\author{
Wei Hua Wang ${ }^{\text {a) }}$ \\ Institute of Physics \& Center for Condensed Matter Physics, Chinese Academy of Sciences, \\ P.O. Box 603, Beijing 100080, People's Republic of China and National Microgravity Laboratory of \\ Chinese Academy of Sciences, Beijing 100080, People's Republic of China \\ Ru Ju Wang and W.T. Yang \\ Institute of Physics \& Center for Condensed Matter Physics, Chinese Academy of Sciences, \\ P.O. Box 603, Beijing 100080, People's Republic of China \\ B.C. Wei \\ National Microgravity Laboratory of Chinese Academy of Sciences, Beijing 10080, \\ People's Republic of China \\ P. Wen, D.Q. Zhao, and M.X. Pan \\ Institute of Physics \& Center for Condensed Matter Physics, Chinese Academy of Sciences, \\ P.O. Box 603, Beijing 100080, People's Republic of China
}

(Received 28 February 2001; accepted 18 March 2002)

\begin{abstract}
The stability of $\mathrm{Zr}_{41} \mathrm{Ti}_{14} \mathrm{Cu}_{12.5} \mathrm{Ni}_{10} \mathrm{Be}_{22.5}$ bulk metallic glass (BMG) upon isothermal annealing near the glass transition temperature has been investigated by using $\mathrm{x}$-ray diffraction, differential scanning calorimetry, and the pulse echo overlap method. The density, elastic constants, and thermodynamic parameters as well as their annealing time dependence have been determined. The microstructural and properties changes of the annealed BMG were checked by acoustic measurement. Obvious structural and property changes were observed with prolonged annealing of the BMG near the glass transition temperature.
\end{abstract}

\section{INTRODUCTION}

Since the discovery of metallic glass in $1960,{ }^{1}$ much research has been devoted to study the formation mechanism and crystallization of metallic glasses. However, the lack of thermal stability in the supercooled liquid of metallic system with respect to crystallization makes the studies of glass transition and nucleation in the metallic alloys very difficult. Recently, a number of multicomponent alloys with high thermal stability of the supercooled liquid against crystallization have been found. The stability has enabled the production of bulk metallic glasses (BMGs) by using various casting processes. ${ }^{2}$ On the other hand, the novel bulk glassy forming liquids can now be studied in much broader time and temperature ranges. The study of stability and nature of the BMG is very important for understanding the excellent glassforming ability (GFA), the crystallization, and the applications of the BMGs. ${ }^{2,3}$ However, the mechanism for the strong stability of the BMGs has not been solved yet. The acoustic method is a powerful tool for studying the structural change and fundamental elastic and thermal behaviors as well as relationship between microstructure and properties. Zr-based BMGs can be produced in a large

a) Address all correspondence to this author.

e-mail: whw@aphy.iphy.ac.cn 3-dimension size, and they show a wide supercooled liquid region. ${ }^{2,3}$ The bulky size of the BMGs is suitable for measurement of elastic wave propagation. The wide supercooled liquid region makes them ideal systems to study the stability and accurately measure structural and physical properties during the prolonged isothermal annealing in the supercooled liquid region. ${ }^{4,5}$ Since the acoustic property is particularly sensitive to the microstructure, ${ }^{6}$ the acoustic velocities can reflect critical information on microstructural evolution and the changes of the physical properties of the BMGs in different states. We show here an ultrasonic investigation on a $\mathrm{Zr}_{41} \mathrm{Ti}_{14} \mathrm{Cu}_{12.5} \mathrm{Ni}_{10} \mathrm{Be}_{22.5} \mathrm{BMG}$ annealed for a prolonged time in its supercooled liquid region. The density and the acoustic velocities upon the annealing time were measured. The elastic constants and thermodynamic parameters are determined from the acoustic data. Marked structural and property changes are observed in the annealed BMG. The stability of the BMG and its relation with GFA and crystallization are discussed.

\section{EXPERIMENTS}

The $\mathrm{Zr}_{41} \mathrm{Ti}_{14} \mathrm{Cu}_{12.5} \mathrm{Ni}_{10} \mathrm{Be}_{22.5}$ ingots were prepared by inductive levitation and melted under a Ti-gettered $\mathrm{Ar}$ atmosphere with oxygen partial pressure of $10^{-9} \mathrm{~Pa}$. The alloy was remelted in a silica tube and quenched in water 
to get a cylindrical rod with $12-\mathrm{mm}$ diameter. ${ }^{7}$ The amorphous nature and the homogeneity were ascertained by x-ray diffraction (XRD), differential scanning calorimetry (DSC), transmission electron microscopy (TEM), and small-angle neutron scattering. ${ }^{7}$ The amorphous rod was cut to a length of $10 \mathrm{~mm}$, and its ends were carefully polished flat and parallel. The sample was annealed in a furnace at $633 \mathrm{~K}$ under vacuum of $2.0 \times 10^{-3} \mathrm{~Pa}$. The annealing temperature is near the glass transition temperature and much lower than the calorimetric crystallization temperature. ${ }^{3}$ The sample was isothermally annealed at various times and cooled to room temperature for the density, XRD, and acoustic measurements. The acoustic velocities were measured at room temperature by using a pulse echo overlap method. ${ }^{4}$ The excitation and detection of the ultrasonic pulses were provided by X- or Y-cut (for longitudinal and transverse waves, respectively) quartz transducers. The frequency of the ultrasonic wave was $10 \mathrm{MHz}$. The travel time of ultrasonic waves propagating through the sample was measured using a MATEC 6600 ultrasonic system with a measuring sensitivity of $0.5 \mathrm{~ns}$. The elastic constants, e.g., bulk modulus $K$, Young's modulus $E$, shear modulus $G$, and Poisson's ratio $\sigma$, and the Debye temperature $\theta_{\mathrm{D}}$ of the BMG are derived from the acoustic velocities and density. ${ }^{4,8}$ Density $\rho$ was measured by the Archimedian principle, and the accuracy was evaluated to be $0.005 \mathrm{~g} / \mathrm{cm}^{3}$ (MAC Science, Japan). XRD was performed using a MAC M03 XHF diffractometer with $\mathrm{Cu} \mathrm{K} \mathrm{K}_{\alpha}$ radiation. DSC measurements were carried out under a purified argon atmosphere in a Perkin-Elmer DSC-7. The Vicher's hardness, $H_{\mathrm{v}}$, was measured with a Newphoto21 microhardness tester at a load of $100 \mathrm{~g}$.

\section{RESULTS AND DISCUSSION}

Figure 1 shows XRD patterns of the BMG annealed at $633 \mathrm{~K}$ for various annealing times. The annealing temperature is near the glass transition temperature $T_{\mathrm{g}}\left(T_{\mathrm{g}}=\right.$ $623 \mathrm{~K}$ ) and much lower than the onset crystallization temperature $T_{\mathrm{x}}\left(T_{\mathrm{x}}=698 \mathrm{~K}\right)$. The XRD pattern for the annealed BMG exhibits no significant difference compared to that of the as-prepared BMG, and no obvious crystalline peaks are observed up to $90 \mathrm{~h}$ annealing in the sample within the examining limit of XRD. However, the positions of the two scattering diffuse peaks obviously shift to higher angle, and the intensity of the scattering peaks also increases with increasing annealing time as indicated in Fig. 1. This phenomenon has not been observed in this BMG by other researchers, because we have more thoroughly studied the structure and properties with increasing annealing time. This suggests that a microstructural change happens when the $\mathrm{BMG}$ is annealed for a prolonged time in the supercooled liquid region, and the annealed BMGs have smaller first nearest-neighbor distance compared with the as-prepared state indicating a close-packed atomic environment. Previous work ${ }^{9-11}$ shows that the BMG separates into two different amorphous phases when annealed in the supercooled liquid region at the earlier stage of the annealing $(0-3 \mathrm{~h})$. The decomposed amorphous phases have different short-range orders and compositions compared with the as-prepared BMG. ${ }^{10}$ The phase separation, which is controlled by atomic mobility, favors the subsequent crystallization and causes the precipitation of nanocrystalline particles in the BMG. The nucleation and growth of $\mathrm{Zr}$-rich crystalline phases ${ }^{9}$ occurs whose compositions are such that they shift the average nearest-neighbor distance of the remaining amorphous alloy by preferentially depleting $\mathrm{Zr}$ with a large atomic volume.

Figure 2 presents continuous DSC traces (with a heating rate of $20 \mathrm{~K} / \mathrm{min}$ ) for the $\mathrm{BMG}$ annealed at $633 \mathrm{~K}$ for various times. The DSC trace of the as-prepared BMG exhibits endothermic characteristics of a glass transition followed by exothermic crystallization reactions at higher temperature. The $T_{\mathrm{g}}, T_{\mathrm{x}}$, and the supercooled liquid region, $\Delta T=T_{\mathrm{x}}-T_{\mathrm{g}}$, are 623,697 , and $74 \mathrm{~K}$, respectively. The first crystallization peak is broadened, and its area (heat release) is decreased with annealing time. After $59 \mathrm{~h}$ of annealing, the first crystallization peak disappears completely, and the position of the second peak shifts to higher temperature with increasing annealing time. Figure 3 shows the changes of $T_{\mathrm{g}}$ and $T_{\mathrm{x}}$ upon annealing time. The value of $T_{\mathrm{g}}$ is monotonically increased with increasing annealing time, and the onset crystallization temperature, $T_{\mathrm{x}}$, is decreased in the earlier annealing stage $(<2 \mathrm{~h})$ and increases monotonically upon annealing after that (as shown in Fig. 3). For annealing

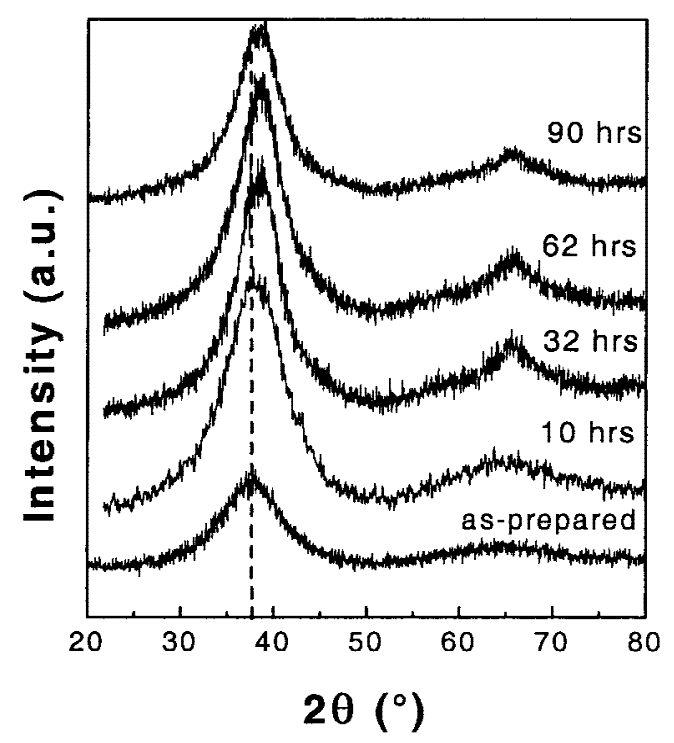

FIG. 1. XRD patterns of the $\mathrm{Zr}_{41} \mathrm{Ti}_{14} \mathrm{Cu}_{12.5} \mathrm{Ni}_{10} \mathrm{Be}_{22.5} \mathrm{BMG}$ at $633 \mathrm{~K}$ for various annealing times. The dash line is a guide for eyes to note the shift of the diffused peak upon annealing time. 
time longer than $59 \mathrm{~h}$, the first crystallization peak disappears. The $\Delta T$ decreases with increase of annealing time, and its midpoint temperature shifts to higher values. The $T_{\mathrm{g}}, T_{\mathrm{x}}$, and $\Delta T$ for the BMG annealed for $90 \mathrm{~h}$ are 668,720 , and $52 \mathrm{~K}$, respectively. The results confirm that the glass transition and the crystallization are significantly affected by annealing. The first crystallization peak is more sensitive to annealing; it shrinks after preannealing and eventually disappears for more than $60 \mathrm{~h}$ annealing. Kinetic analysis ${ }^{13}$ indicates that the first crystallization reaction has a much smaller apparent

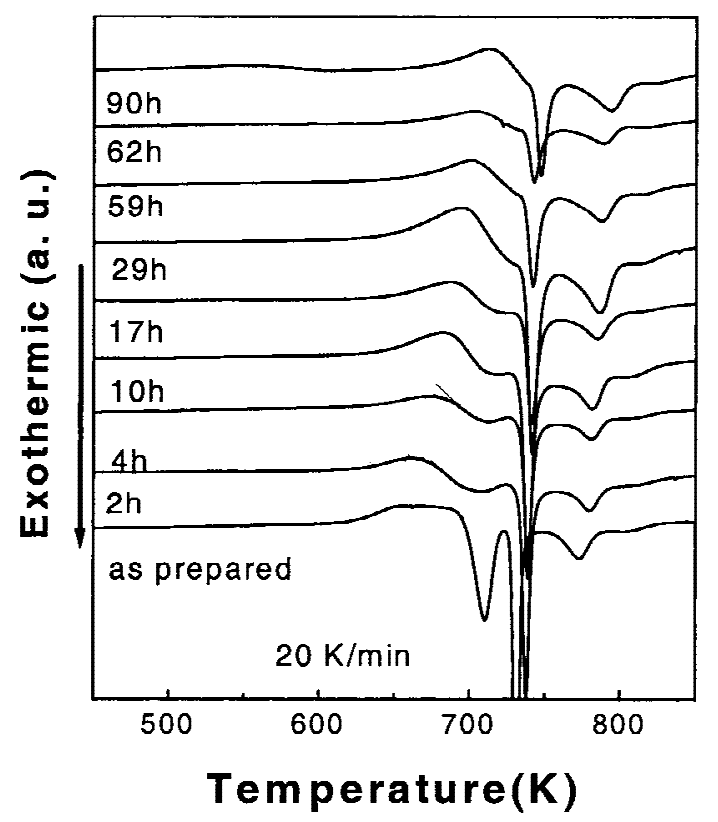

FIG. 2. DSC traces of the $\mathrm{Zr}_{41} \mathrm{Ti}_{14} \mathrm{Cu}_{12.5} \mathrm{Ni}_{10} \mathrm{Be}_{22.5} \mathrm{BMG}$ at $633 \mathrm{~K}$ for various annealing times.

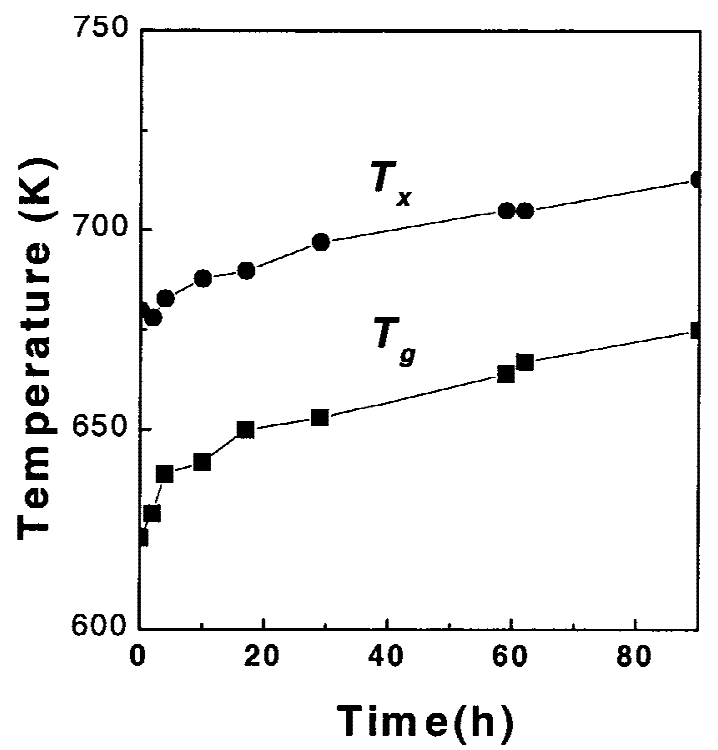

FIG. 3. Change of the $T_{\mathrm{g}}$ and $T_{\mathrm{x}}$ of the $\mathrm{Zr}_{41} \mathrm{Ti}_{14} \mathrm{Cu}_{12.5} \mathrm{Ni}_{10} \mathrm{Be}_{22.5} \mathrm{BMG}$ annealed at $633 \mathrm{~K}$ for various annealing times. activation energy comparing with the second crystallization peaks. This is the reason the first crystallization peak is so sensitive to the annealing. Previous work found that the first crystallization was also sensitive to the small elements addition, high heating rate, and high pressure which confirms the above results. ${ }^{7,13}$ The DSC trace, which is not plotted, for the sample annealed for 40 min shows only slight difference compared with that of the asprepared sample indicating that the microstructural change in the BMG needs some incubation time. The DSC results demonstrate that the primary crystallization induced by isothermal annealing occurs near $T_{\mathrm{g}}$, but the crystalline particles are too small to be detected by XRD. However, the primary crystallization had been observed by high-resolution TEM. ${ }^{9}$ Schneider et al. ${ }^{9}$ proposed that the primary nanocrystallization was preceded by a modulated chemical decomposition process. The isoannealing causes structural change in the BMG, and the structural change affects markedly the subsequent glass transition and crystallization behavior. From DSC curves, the total crystallization enthalpy, $\Delta H_{\mathrm{c}}(t)$, can be calculated and the crystallization volume fraction, $V_{\mathrm{c}}(t)$, can be estimated according to the formulas of $V_{\mathrm{c}}(t)=\left[\Delta H_{\mathrm{c}}(0)-\right.$ $\left.\Delta H_{\mathrm{c}}(t)\right] / \Delta H_{\mathrm{c}}(0)$. Figure 4 exhibits the change of $\Delta H_{\mathrm{c}}(t)$ and $V_{\mathrm{c}}(t)$ upon annealing time, t. It can be clearly seen that the $\Delta H_{\mathrm{c}}(t)$ decreases upon annealing time, while the $V_{\mathrm{c}}(t)$ increases with $\mathrm{t}$ to $32 \%$ after annealing for $90 \mathrm{~h}$.

To check the microstructural change and the influence of the primary crystallization on the properties of the annealed alloy, acoustic measurements were performed on the annealing samples at room temperature. The values of $\rho$, longitudinal velocity $v_{1}$, shear velocity $\nu_{\mathrm{s}}$, and $\mathrm{H}_{\nu}$ of the as-prepared BMG are $6.108 \mathrm{~g} / \mathrm{cm}^{3}, 5.13 \mathrm{~km} / \mathrm{s}$, $2.43 \mathrm{~km} / \mathrm{s}$, and $5.9 \mathrm{GPa}$, respectively. The $E, G, K, \sigma$, and $\theta_{\mathrm{D}}$ calculated from the acoustic data are $97.7 \mathrm{GPa}, 36.0$ $\mathrm{GPa}, 113.0 \mathrm{GPa}, 0.356$, and $321.0 \mathrm{~K}$, respectively. The obtained elastic data for the as-prepared BMG are in good agreement with the data measured using different

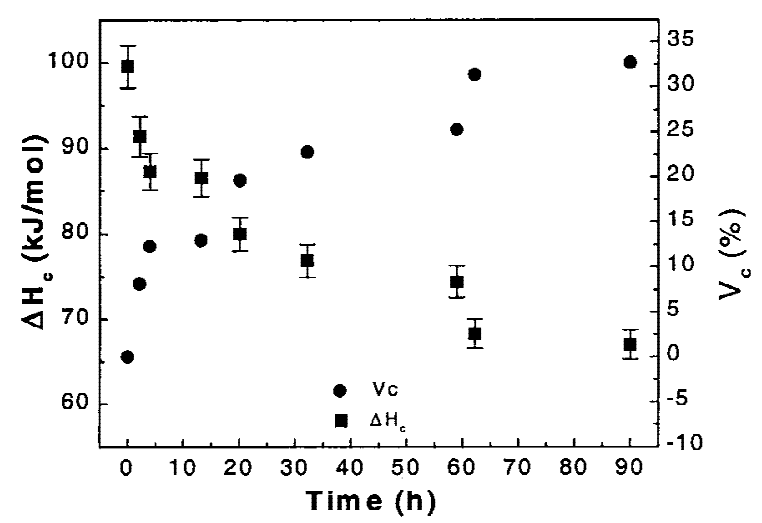

FIG. 4. Changes of crystallization enthalpy $\Delta H_{\mathrm{c}}(t)$ and crystalline phase volume fraction $V_{c}(t)$ of the $\mathrm{Zr}_{41} \mathrm{Ti}_{14} \mathrm{Cu}_{12.5} \mathrm{Ni}_{10} \mathrm{Be}_{22.5} \mathrm{BMG}$ annealed at $633 \mathrm{~K}$ for various annealing times. 
methods. ${ }^{14}$ This confirms that the ultrasonic methods can provide exact information about elastic properties of the BMG. Figure 5 presents the variations $\Delta Y / Y_{0}=[Y-$ $\left.Y_{0}\right] / Y_{0}$ of the $\nu_{1}, \nu_{\mathrm{s}}$, and density of the BMG annealed at $633 \mathrm{~K}$ for various times. Both $\nu_{1}$ and $\nu_{\mathrm{s}}$ increase with increasing annealing time as indicated in Fig. 5(a). The change of $v_{\mathrm{s}}$ upon annealing time is much larger than that of $\nu_{1}$. The large density difference, $1 \%$ (the relative density change between as-prepared BMG and fully crystallized BMG is about $1.2 \%{ }^{5}$ ), can be seen in Fig. 5(b). The result is in agreement with that of XRD and demonstrates the densification of the structure with increasing annealing time. The corresponding elastic constants changes of $Y(Y=E, G, K)$ of the BMG calculated from the velocities are shown in Fig. $6 . Y$ is normalized by $\Delta Y / Y_{0}=\left(Y-Y_{0}\right) / Y_{0}$, where $Y_{0}$ is the normal modulus for the as-prepared BMG. The $E, G$, and $K$ show similar change trends with increasing time. Large changes in the $v_{\mathrm{s}}(9.6 \%), \theta_{\mathrm{D}}(9.6 \%), G(21.4 \%), \rho(1.0 \%)$, and $\nu_{1}(3.2 \%)$ between the BMG in as-prepared and 90-h annealed states have been induced. The marked properties change during the prolonged annealing process confirms the microstructural change induced by isothermal annealing near $T_{\mathrm{g}}$. In fact, the glass-matrix composite containing nanocrystalline particles is formed in the annealing process; the composite has different acoustic and elastic properties.

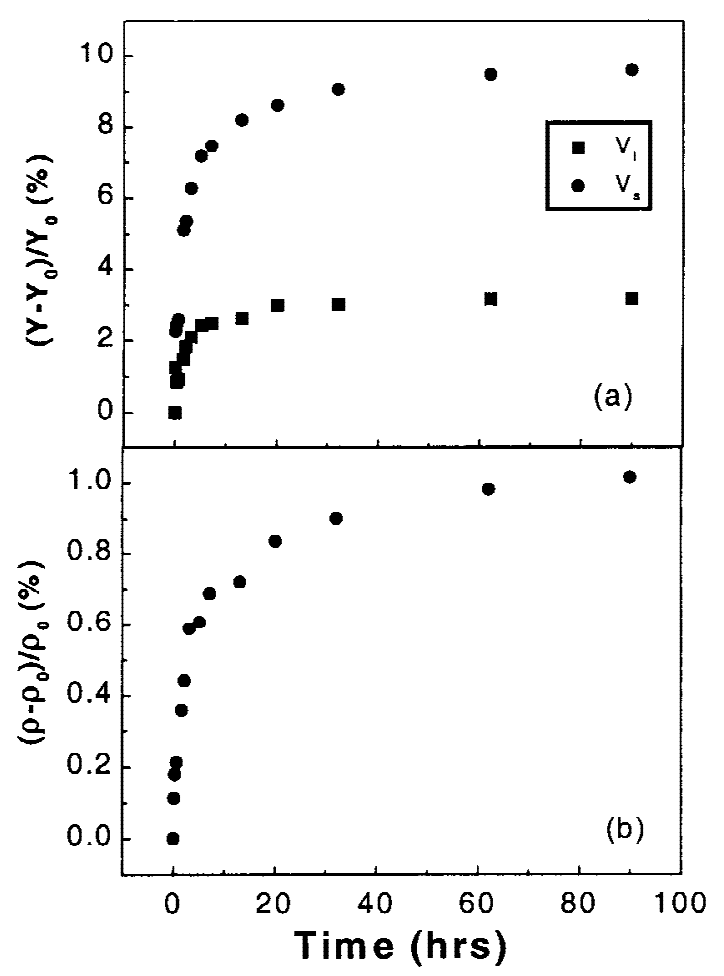

FIG. 5. Relative (a) longitudinal, transverse velocities and (b) density changes upon annealing time at $633 \mathrm{~K}\left(Y=\rho, v_{1}\right.$, and $\left.v_{\mathrm{s}}\right) . Y$ is normalized by $\Delta Y / Y_{0}=\left(Y-Y_{0}\right) / Y_{0}$, where $Y_{0}$ is a corresponding value for the as-prepared BMG.
Primary nanocrystallization, ${ }^{9}$ phase separation, ${ }^{9-10,15}$ and local microstructural change from short-range order to medium-range order ${ }^{16}$ depending on annealing conditions have been observed in the supercooled liquid region of the BMG. The large density difference (the relative density change is about $1.0 \%$ ), elastic constants changes, and XRD and DSC results provide additional evidence that nanocrystalline precipitation occurs in the BMG reheated near to $T_{\mathrm{g}}$. The nanocrystalline particles cannot be detected by XRD indicating that the growth of the nuclei is inhibited in this annealing condition; this is due to the diffusion limited growth in the very alloy in supercooled liquid state. We choose the property changes of $G$ and $E$, which are structure-sensitive, and $V_{\mathrm{c}}$ to plot versus $\ln (t)$. Figure 7 shows the plot of $\Delta Y / Y_{0}$ versus $\ln (t)$. It can be seen from Fig. 7 that the change of the measured $G, E$, and $V_{\mathrm{c}}(t)$ of the BMG varies roughly linearly with the $\ln (t)$ and an approximate relation $\Delta Y / Y_{0} \infty \ln (v t)$, where $v$ is the attempt frequency, to be obeyed in the isothermal annealing; this corresponds to a continuous spectrum of activation energies for the relaxation in the BMG. ${ }^{17-20}$

Our experimental results indicate that the $\mathrm{ZrTiCuNiBe}$ BMG has following characteristics: (i) It has a wide supercooled liquid region with sluggish kinetics resulting in strong resistance against crystallization. The diffusion limited growth and high nucleation rate in the supercooled liquid region lead to nanocrystallization. The alloy has five metallic components with a large atomic-size mismatch and a composition close to a deep eutectic; ${ }^{3}$ its supercooled liquid is then a highly dense liquid with

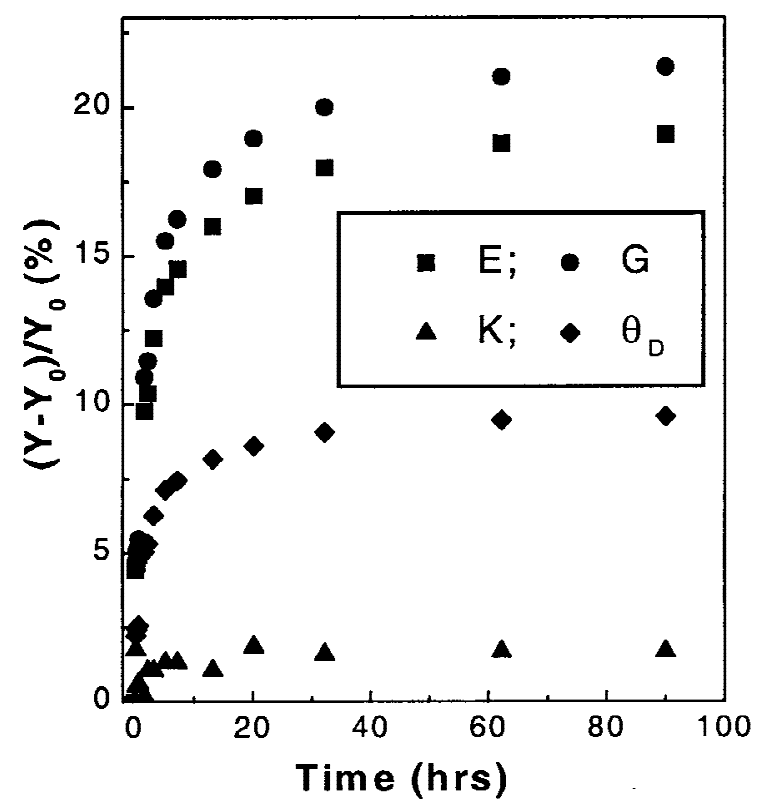

FIG. 6. Relative changes $\Delta Y / Y_{0}=\left(Y-Y_{0}\right) / Y_{0}$ of variation of elastic constants and Debye temperature $E, G, K$, and $\theta_{\mathrm{D}}(Y=E, G, K$, and $\left.\theta_{\mathrm{D}}\right)$ of the BMG with increasing annealing time. A large relative change of $G, E$, and $\theta_{\mathrm{D}}$ and a smaller change of $K$ in the 90-h-annealed sample relative to the as-prepared glassy state can be clearly seen. 


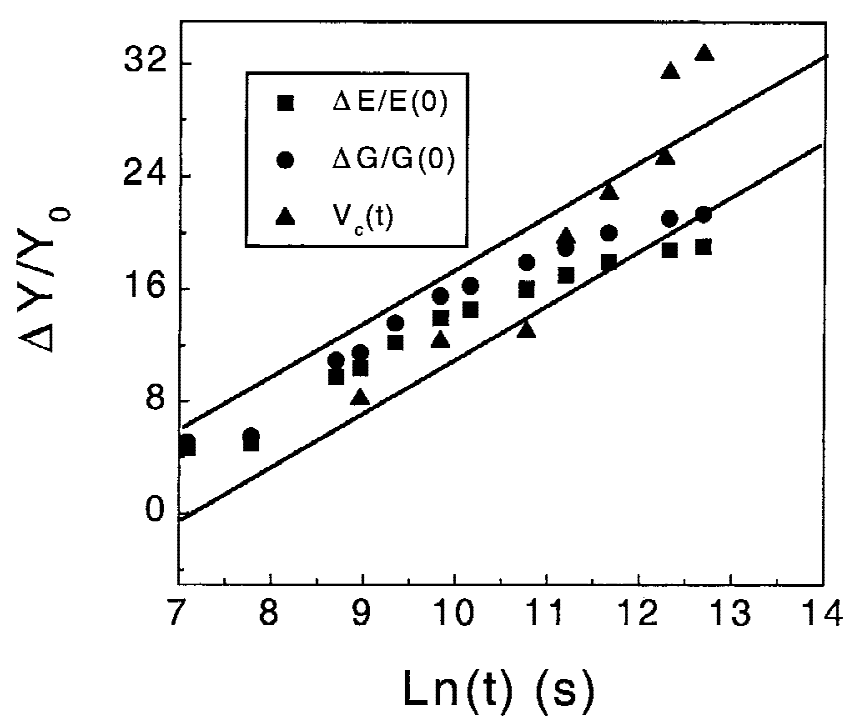

FIG. 7. Relative changes $\Delta Y / Y_{0}\left(Y=E, G\right.$, and $\left.V_{\mathrm{c}}\right)$ of the BMG with the logarithm of the isothermal annealing time $\ln (t)$. The solid lines in the figure are for guiding the eyes.

small free volumes (about $1 \%^{21}$ ) and large viscosity that are several orders of magnitude higher than those of previously known alloys. ${ }^{22}$ In addition, the melt is energetically close to its corresponding crystalline state, ${ }^{23}$ and the driving force for crystallization is relatively small. These factors result in a strong liquid behavior and sluggish crystallization kinetics and lead to the wide supercooled liquid region reflecting a very low critical cooling rate for the metallic glass formation or excellent glassforming ability. (ii) The nanocrystallization obeys the "ln $(t)$ " kinetic ${ }^{18-20}$ corresponding to a continuous spectrum of activation energies for the atomic rearrangement in the BMG. (iii) The BMG can be transformed into a glass-matrix composite containing nanocrystalline particles when it is annealed for a prolonged time much below the crystallization temperature.

\section{CONCLUSIONS}

Primary crystallization occurs in the $\mathrm{Zr}_{41} \mathrm{Ti}_{14^{-}}$ $\mathrm{Cu}_{12.5} \mathrm{Ni}_{10} \mathrm{Be}_{22.5} \mathrm{BMG}$ when it is annealed for prolonged time near the glass transition temperature. The isothermal annealing near $T_{\mathrm{g}}$ leads to primary nanocrystallization which obeys the " $\ln (t)$ " kinetic. Obvious structural and property changes are observed in the BMG reheated into the supercooled liquid state. The BMG can be transformed into a glass-matrix composite containing nanocrystalline particles.

\section{ACKNOWLEDGMENT}

The authors are grateful for the financial support of the National Natural Science Foundation of China (Grant Nos. 50031010 and 59925101).

\section{REFERENCES}

1. W. Klement, R. Willens, and P. Duwez, Nature 187, 869 (1960).

2. A. Inoue, Mater. Trans. JIM 36, 866 (1995).

3. W.L. Johnson, Mater. Science Forum 225-227, 35 (1996).

4. W.H. Wang, H.Y. Bai, J.L. Luo, R.J. Wang, and J. Duo, Phys. Rev. B 62, 25 (2000).

5. W.H. Wang, R.J. Wang, F.Y. Li, and M.X. Pan, Appl. Phys. Lett. 74, 1803 (1999).

6. D. Schreiber, Elastic Constants and Their Measurement (McGrawHill, New York, 1973).

7. W.H. Wang and H.Y. Bai, J. Appl. Phys. 84, 5961 (1998).

8. L.A. Girifalco, Statistical Physical of Materials (Wiley, New York, 1973), p. 78.

9. S. Schneider, P. Thiyagarajan, and W.L. Johnson, Appl. Phys. Lett. 68, 493 (1996).

10. W.H. Wang, Q. Wei, and S. Friedrich, Phys. Rev. B 57, 8211 (1998).

11. J.M. Liu, M.H. Macht, and A. Wiedemann, Mater. Sci. Eng. A 222, 182 (1997).

12. Y.X. Zhuang, W.H. Wang, M.X. Pan, and D.Q. Zhao, Appl. Phys. Lett. 75, 2392 (1999).

13. W.H. Wang, Y.X. Zhuang, M.X. Pan, and Y.S. Yao, J. Appl. Phys. 88, 3914 (2000).

14. R.D. Conner, R.B. Dandliker, and W.L. Johnson, Acta Mater. 46, 6089 (1998).

15. R. Busch, S. Schneider, A. Peker, and W.L. Johnson, Appl. Phys. Lett. 67, 1544 (1995).

16. W.H. Wang, R.J. Wang, M.X. Pan, and Y.S. Yao, Phys. Rev. B 62, 11292 (2000).

17. K. Bothe and H. Neuhaeuser, Scr. Metall. 16, 1053 (1982).

18. W. Primak, Phys. Rev. 100, 1677 (1955).

19. M.R.J. Gibbs, J.E. Evetts, and J.A. Leake, J. Mater. Sci. 18, 278 (1983).

20. V.A. Khonik, Phys. Status Solidi A 177, 173 (2000).

21. K. Samwer, R. Busch, and W.L. Johnson, Phys. Rev. Lett. 2, 580 (1999).

22. E. Bakke, R. Busch, and W.L. Johnson, Appl. Phys. Lett. 67, 3260 (1995).

23. R. Busch, W. Liu, and W.L. Johnson, J. Appl. Phys. 83, 4134 (1998). 